\title{
A bounding scheme for proving the Wright con- jecture on delay differential equations
}

\author{
Balázs Bánhelyi ${ }^{1}$, Tibor Csendes ${ }^{1 *}$, Tibor Krisztin ${ }^{2}$, and Arnold \\ Neumaier ${ }^{3}$ \\ ${ }^{1}$ Institute of Informatics, University of Szeged, Árpád tér 2, 6720 Szeged, Hungary, \\ banhelyi@inf.szte.hu, csendes@inf.szte.hu \\ ${ }^{2}$ Bolyai Institute, University of Szeged, Aradi vértanúk tere 1, 6720 Szeged, Hun- \\ gary, krisztin@math.u-szeged.hu \\ ${ }^{3}$ Department of Mathematics, University of Vienna, A-1090 Vienna, Austria, \\ arnold.neumaier@univie.ac.at \\ * Corresponding author
}

Abstract: We provide here an elementary derivation of the bounding scheme applied for proving the Wright conjecture on delay differential equations. We also report a minor extension of the parameter range where the conjecture was proven, to $\alpha \in[1.5,1.57065]$.

Keywords: bounding scheme; delay differential equation; interval arithmetic; validated computation; Wright conjecture

\section{Introduction}

In this paper we give an elementary derivation of a bounding scheme to prove Wright's conjecture [6] on the delay differential equation

$\dot{u}(t)=-\alpha u(t-1)[1+u(t)], \quad \alpha>0$.

That bounding scheme is then applied in a verified computational algorithm for systematic checking the $\alpha$ values in question. If we consider only those solutions of equation (1) which have values in $(-1, \infty)$, the transformation $x=\log (1+u)$ leads to the equation

$\dot{x}(t)=f_{\alpha}(x(t-1))$

with $f_{\alpha}(\xi)=-\alpha\left(e^{\xi}-1\right), \xi \in \mathbb{R}$. Throughout this paper (2) will also be called Wright's equation.

In [2] we proved 
Theorem 1. If $\alpha \in[1.5,1.5706]$, then the zero solution of equation (2) is globally attractive.

We used the following statement in the proof:

Theorem 2. The zero solution of (2) is globally attracting if and only if (2) has no slowly oscillating periodic solution.

Recall that a solution $x: R \rightarrow R$ oscillates slowly if $\left|z_{1}-z_{2}\right|>1$ for any two different zeros of $x$. In [2] a theoretical proof was given for

Corollary 1. If $0<\alpha<\frac{\pi}{2}$ and $p^{\alpha}: \mathbb{R} \rightarrow \mathbb{R}$ is a slowly oscillating periodic solution of equation (2) then

$$
\max _{t \in \mathbb{R}} p^{\alpha}(t) \geq \log \frac{\pi}{2 \alpha}>1-\frac{2 \alpha}{\pi} .
$$

The computational part of the proof of Theorem 1 proves

Theorem 3. If $\alpha \in[1.5,1.5706]$ and $y: \mathbb{R} \rightarrow \mathbb{R}$ is a slowly oscillating periodic solution of (2), then $\max _{t \in \mathbb{R}}|y(t)| \leq 1-\frac{2 \alpha}{\pi}$.

Now, a combination of Theorem 2, Corollary 1, and Theorem 3 proves Theorem 1.

In an earlier paper [1], the first author investigated the problem with traditional verified differential equation solver algorithms $[4,5]$. He found that a proof of the conjecture along these lines would require an enormous amount of computation time with the present technological conditions (compilers, algorithms and computer capacities). He was able to prove only that for all $\alpha$ values within the tiny interval $\left[1.5,1.5+10^{-22}\right]$ the trajectories of the solutions will reach a phase when the absolute value of the solution remain below 0.075 for a time interval of a unit length. For wider parameter intervals, or for values closer to $\pi / 2$ the required CPU times exploded. Thus traditional computer-assisted techniques involving general, inclusion monotone iterative techniques for differential equations appear not suitable for settling the conjecture.

\section{The bounding scheme}

Let $p: \mathbb{R} \rightarrow \mathbb{R}$ be a nontrivial periodic solution of (2). Set $M=\max _{t \in \mathbb{R}} p(t)$ and $-m=\min _{t \in \mathbb{R}} p(t)$. We skip here the technical details from Wright's paper, and just give the conditions obtained by him:

$M \leq-\alpha\left(e^{-m}-1\right)+(-m) \frac{e^{-m}}{e^{-m}-1}-1$ if $\alpha\left(e^{-m}-1\right) \leq-m$,

$M \leq \alpha-\frac{1-e^{\alpha\left(e^{-m}-1\right)}}{\left(1-e^{-m}\right)}$, 
$m \leq \alpha\left(e^{M}-1\right)-M \frac{e^{M}}{e^{M}-1}+1$

The present approach follows another line of thought, still it is a kind of direct extension of that of Wright. Denote three subsequent zeroes of the trajectory by 0 , $z_{1}$, and $z_{2}$. We may assume that $y(t)>0$ for $t \in\left(0, z_{1}\right)$, and $y(t)<0$ for $t \in\left(z_{1}, z_{2}\right)$. Let us define the following functions bounding the trajectories (see Figure 1):

$y_{(\text {inc, }, 1)}^{(\text {upper })}(t)$ : an upper bounding function for the time interval $0 \leq t \leq 1$,

$y_{(\text {inc }, 1)}^{(\text {lower })}(t)$ : a lower bounding function for the time interval $0 \leq t \leq 1$,

$y_{(\text {dec }, n)}^{(\text {upper })}(t):$ an upper bounding function for the time interval $1 \leq t \leq z_{1}$,

$y_{(\text {dec }, 1)}^{(\text {lowe })}(t)$ : a lower bounding function for the time interval $z_{1} \leq t \leq z_{1}+1$,

$y_{(\text {dec }, 1)}^{(\text {upper })}(t)$ : an upper bounding function for the time interval $z_{1} \leq t \leq z_{1}+1$,

$y_{(\text {inc, }, n)}^{(\text {lower })}(t)$ : a lower bounding function for the time interval $z_{1}+1 \leq t \leq z_{2}$.

The trajectory bounding functions are illustrated by dashed lines on Figure 1. Here four consecutive time intervals will be considered defined by the zeros and by the extremal values of the trajectory denoted by $($ inc, 1$),($ dec,$n),($ dec, 1$)$, and $(i n c, n)$, respectively. The length of the time intervals $(i n c, 1)$ and $($ dec, 1$)$ are known to be one. On the other hand the length of $(\operatorname{dec}, n)$, denoted as $p_{M}=z_{1}-1$ and that of (inc, $n), p_{m}=z_{2}-z_{1}-1$ are unknown, it is even unclear whether these are larger than one.

The trajectory bounding functions will be sharpened sequentially, in an iterative way, i.e. the bounding functions of the time interval $(i n c, 1)$ will be used to improve the bounding function on the interval $(d e c, n)$, etc. Then, the bounding function of the last interval, $($ inc,$n)$ will be used to make the inequalities for the interval (inc, 1$)$ sharper, and so on. Those bounding function improvements that are based on a single bounding function of the earlier time interval are basically similar to the original technique used by Wright. The sharpening steps using two bounding functions on the argument interval apply a new, Taylor series based method to be described later in this paper. At start we set the upper bounding functions to constant $M$, the lower bounding functions to $-m$ with the exceptions of $y_{(\text {inc, }, 1)}^{(\text {lower })}=0$ and $y_{(\text {dec }, 1)}^{(\text {upper })}=0$.

We iterate only on such cases, when the conditions (3) to (5) and that of Corollary 1 are fulfilled. The conditions we check at the end of each iteration cycle of the 


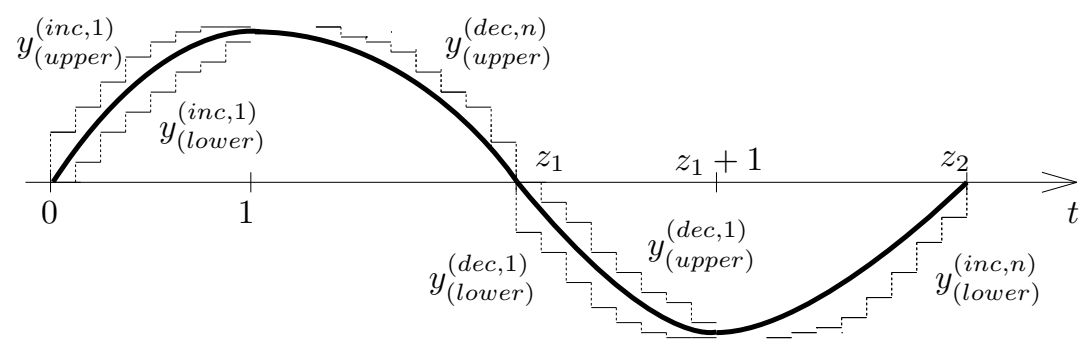

Figure 1

The trajectory bounding functions shown as dashed lines for a full period

bounding function sharpening procedure are

$y_{(\text {inc }, 1)}^{\text {(upper })}(0+1)<M \quad$ and $\quad-m<y_{(\text {dec }, 1)}^{\text {(lower })}\left(z_{1}+1\right)$.

In case at least one of these conditions are satisfied then the solution of the investigated delay differential equation cannot have a periodic solution with a maximal value of $M$ and the minimal value of $m$ as assumed for the given $\alpha$ parameter.

\section{Improved bounds for the unit width intervals}

First we show how to obtain an upper bound on the periodic trajectory on the interval $($ inc, 1$)$ based on the $y_{(\text {inc, }, n)}^{(\text {lower })}(t)$ function. Since $y_{(\text {inc }, n)}^{(\text {lower })}(t)$ is a lower bounding function, so $y_{(\text {inc }, n)}^{(\text {lower })}(t) \leq y(t)$ holds for all $t \leq 0$. Now integrate $y^{\prime}$ from 0 to $t$ $(0 \leq t \leq 1)$ :

$$
\begin{aligned}
y(t)= & y(t)-y(0)= \\
& -\alpha \int_{0}^{t} e^{y(x-1)}-1 d x=-\alpha \int_{0-1}^{t-1} e^{y(x)}-1 d x \leq-\alpha \int_{0-1}^{t-1} e^{y_{(\text {inc }, n)}^{(\text {lower })}(x)}-1 d x .
\end{aligned}
$$

We can obtain a new, stronger bounding function from this bound and from the old one for the $t \geq 0$ case:

$$
y_{(\text {inc }, 1)}^{(\text {upper })}(t)=\min \left\{\begin{array}{c}
y_{(\text {inc }, 1)}^{\text {(upper })}(t) \\
-\alpha \int_{0-1}^{t-1} e^{y_{(\text {inc }, n)}^{(\text {lower })}(x)}-1 d x
\end{array}\right\}, t \in[0,1] .
$$

We suppress the iteration number in the bounding function, the new one on the left hand side of the defining equation is calculated from the old function on the right 
hand side as it is usual in computer programs. We can get a new bounding function for the lower bounding function in $(d e c, 1)$ in a similar way:

$$
y_{(\text {dec }, 1)}^{(\text {lowe })}(t)=\max \left\{\begin{array}{c}
y_{(\text {dec }, 1)}^{(\text {lower })}(t) \\
-\alpha \int_{z_{1}-1}^{t-1} e^{y_{(\text {dec }, n)}^{(\text {upper })}(x)}-1 d x
\end{array}\right\}, t \in\left[z_{1}, z_{1}+1\right] .
$$

We can obtain an improved lower bound for the trajectory on the interval $($ inc, 1$)$ by $y(1)-y(t)=M-y(t)=$

$$
-\alpha \int_{t}^{1} e^{y(x-1)}-1 d x=-\alpha \int_{t-1}^{0} e^{y(x)}-1 d x \leq-\alpha \int_{t-1}^{0} e^{y_{(\text {inc }, n)}^{(\text {lower })}(x)}-1 d x .
$$

The new lower bounding function is then

$$
y_{(\text {inc }, 1)}^{(\text {lowe })}(t)=\max \left\{\begin{array}{c}
y_{(\text {inc }, 1)}^{(\text {lower })}(t) \\
M+\alpha \int_{t-1}^{0} e^{y_{(\text {inc }, n)}^{(\text {lower })}(x)}-1 d x
\end{array}\right\} \text { if } t \in[0,1] .
$$

We can build an improved upper bound also for the time interval $(\mathrm{dec}, 1)$ in a similar way:

$$
y_{(\text {dec }, 1)}^{(\text {upper })}(t)=\min \left\{\begin{array}{c}
y_{(\text {dec }, 1)}^{(\text {upper })}(t) \\
-m+\alpha \int_{t-1}^{0} e^{y_{(\text {dec }, n)}^{(\text {uppr })}(x)}-1 d x
\end{array}\right\} \text { if } t \in[0,1] .
$$

By that we have completed the description of the improved bounding functions for the unit width time intervals.

\section{Bounds for the period length}

A sharp enclosure of the period length is very important for the success of the proof for the conjecture, especially for $\alpha$ values close to $\pi / 2$. To calculate bounds on the period length and as a part of that bounds for the not unit length time intervals we apply an Euler type differential equation solution method

$$
\begin{gathered}
Y(x)=Y\left(x_{0}\right)+Y^{(1)}\left(\left[x_{0}, x\right]\right)\left(x-x_{0}\right), \\
Y\left(\left[x_{0}, x\right]\right)=Y\left(x_{0}\right)+Y^{(1)}\left(\left[x_{0}, x\right]\right)\left(\left[0, x-x_{0}\right]\right)
\end{gathered}
$$

customized for delay equations. In these equations we used the notions of interval calculations [5], i.e. capitals denote interval values. The implementation details will 


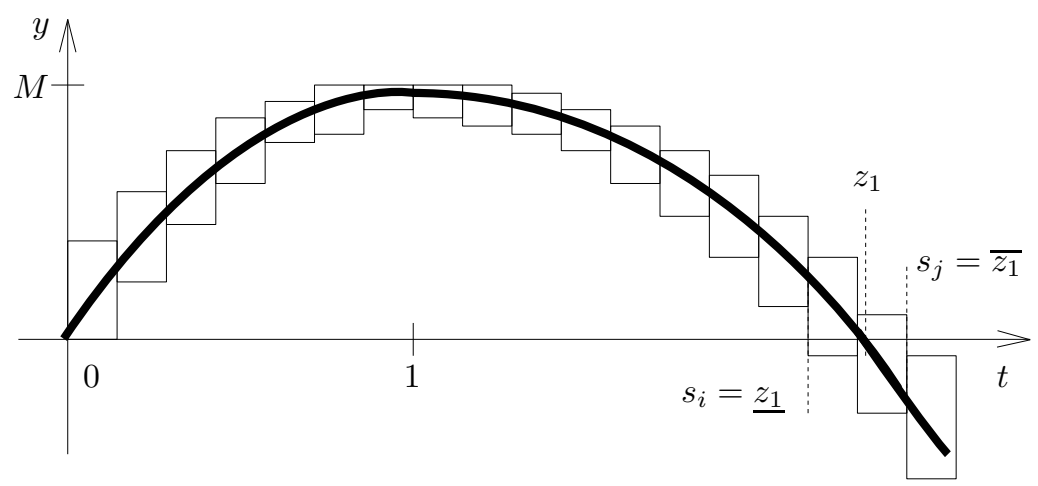

Figure 2

Illustration of the bounding procedure for the $z_{1}$ zero of the trajectory

be discussed in the next section. To use this method we need an enclosure $Y\left(x_{0}\right)$ of the trajectory in the start point, and bounds on a given number of time intervals covering together unit length time intervals.

For these calculations we need lower and upper bounds for the trajectory on the unit length time intervals before the investigated $(d e c, n)$ and $(i n c, n)$ phases. These are available due to the previous subsection. The lower and upper bounds for the zeros $z_{1}$ and $z_{2}$ of the trajectory will be determined using the interval enclosures obtained on time intervals for the trajectory. Consider first the case when we follow the trajectory from 1 to find $z_{1}$, i.e. we want to find bounds for $p_{M}$. Assume that as a part of the verified integration the first interval that contains zero is $Y\left(t_{i}, t_{i}+h\right)$, where $h$ is the step size of the numerical integration. Then there may follow some integration steps for which the respective $Y$ enclosures contain zero. Let the last such be $Y\left(t_{j}, t_{j}+h\right)$ (in some cases it is possible that $i=j$ ). Then $\left[t_{i}, t_{j}+h\right]$ is obviously a verified enclosing interval for $z_{1}$. The same technique that is illustrated on Figure 2 is also applicable for the bounding of $p_{m}$.

Denote the enclosures of $p_{M}$ and $p_{m}$ to be calculated from the above bounds of the zeros by $P_{M}$ and $P_{m}$, respectively. The lower and upper bounds of these intervals are denoted as usual in interval calculation, with underline and overline, e.g. $P_{M}=$ $\left[\underline{P}_{M}, \bar{P}_{M}\right]$.

\section{Improved bounds for the not unit width intervals}

As we could see in the previous subsection, it is not easy to determine $z_{1}$, as the zero of the investigated trajectory. In the present subsection we build a valid upper bound for the trajectory on the intervals $(i n c, 1)$ and $(d e c, n)$ that can be applied as needed also until the point $z_{1}$ for calculating further improving bounds on the interval $(\mathrm{dec}, 1)$. 
Consider the trajectory on $\left[0,1+\bar{P}_{M}\right]$, i.e. on the intervals $($ inc, 1$)$ and $(\operatorname{dec}, n)$. The bounds on the trajectory are at this point obtained by the new bounds of (9) and (10) on (inc, 1$)$, and by the verified solution of the differential equation, as described in Section 4 on $(\operatorname{dec}, n)$. Let us call this complete bounding function as $Y$, and its upper bound as $\bar{Y}$. For a monotonically increasing $y(t)$ function we have

$$
y(t) \geq y(t-\Delta t) \text { if } \Delta t \geq 0
$$

and for a monotonically decreasing $y(t)$ function

$$
y(t) \geq y(t-\Delta t) \text { if } \Delta t \leq 0 .
$$

The trajectory is known to be strictly monotonically increasing on (inc, 1$)$, while strictly monotonically decreasing on $(\operatorname{dec}, n)$.

Consider first the $($ inc, 1$)$ time interval, here the $y_{(\text {inc,1) }}^{(\text {upper })}$ gives an upper bounding function, $\bar{Y}$ for the periodic trajectory. Since $p_{M} \leq \bar{P}_{M}$, the relation

$$
\Delta t=\left(1+\bar{P}_{M}\right)-z_{1}=\bar{P}_{M}-p_{M} \geq 0
$$

holds. Now these imply

$$
\bar{Y}(t) \geq y(t) \geq y(t-\Delta t)=y\left(t-\left(\left(1+\bar{P}_{M}\right)-z_{1}\right)\right) .
$$

These relations can be interpreted as $\bar{Y}$ is an upper bounding function also for $y(t-$ $\Delta t)$, i.e. for the trajectory shifted by $\Delta t$ on the interval

$$
\begin{gathered}
{\left[-\left(\left(1+\bar{P}_{M}\right)-z_{1}\right), 1-\left(\left(1+\bar{P}_{M}\right)-z_{1}\right)\right]=} \\
{\left[-\left(\bar{P}_{M}-p_{M}\right), 1-\left(\bar{P}_{M}-p_{M}\right)\right]=\left[z_{1}-\bar{P}_{M}-1, z_{1}-\bar{P}_{M}\right] .}
\end{gathered}
$$

Consider now the $(d e c, n)$ phase, the verified solution will give an upper bound for $y(t)$ on $\left[1,1+\underline{P}_{M}\right]$. Here $y(t)$ is strictly monotonically decreasing, thus due to $\underline{P}_{M} \leq p_{M}$ the relations

$$
\bar{Y}(t) \geq y(t) \geq y(t-\Delta t)=y\left(t-\left(\left(1+\underline{P}_{M}\right)-z_{1}\right)\right)
$$

hold with $\Delta t=\underline{P}_{M}-p_{M} \leq 0$. Here again $\bar{Y}$ is an upper bounding function also for $y(t-\Delta t)$, i.e. for the trajectory shifted by $\Delta t$ on the interval

$$
\begin{gathered}
{\left[1-\left(\underline{P}_{M}-p_{M}\right), 1+\underline{P}_{M}-\left(\underline{P}_{M}-p_{M}\right)\right]=} \\
{\left[z_{1}-\underline{P}_{M}, z_{1}\right] .}
\end{gathered}
$$

The explanation for the above bounding technique is illustrated on Figure 3. The first case can be understood as if the original periodic solution would be shifted in such a way that the original $z_{1}$ zero coincides with $1+\bar{P}_{M}$. Since $y(t)$ is monotonically increasing on the interval (inc, 1$)$, thus the upper bounding function $\bar{Y}(t)$ remains an upper bound of the shifted function too (upper picture of Figure 3). The 
highlighted upper bounding functions parts are presented as bounds of the $y(t)$ trajectory.

In the second case the original trajectory is shifted in such a way that the zero $z_{1}$ coincides with $\left(1+\underline{P}_{M}\right)$. The monotonically decreasing $y(t)$ will then remain below $\bar{Y}(t)$ on the given time interval (see the second picture of Figure 3). As it can be seen on this figure, in the gap between the two highlighted function we consider the constant $M$ value. With the above considerations we have provided a bounding function that can be used also until the unknown $z_{1}$ time point.

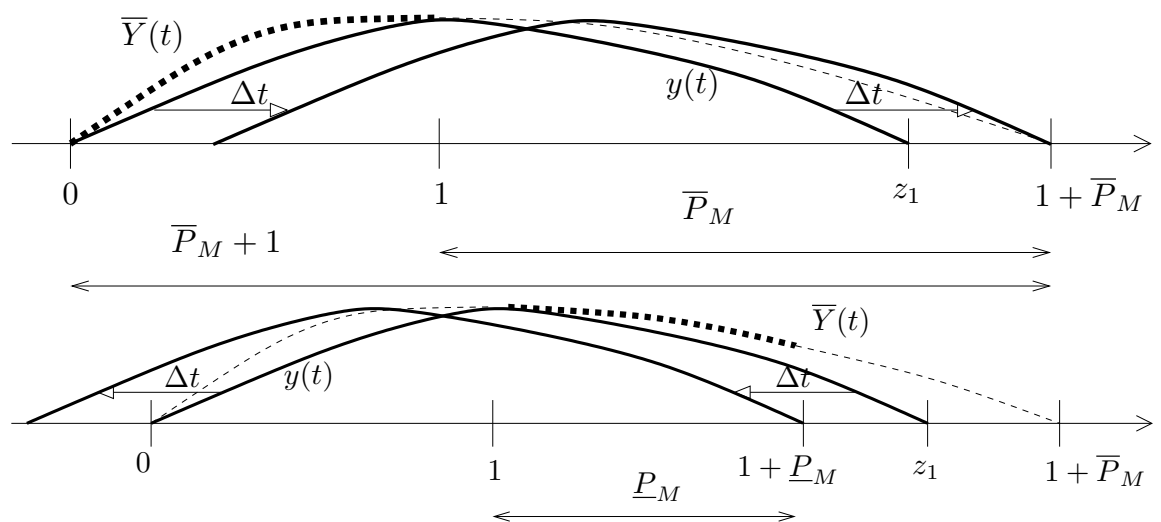

Figure 3

Illustrations of how the bounds can be obtained for the cases when the shifted $z_{1}$ coincides with $1+\bar{P}_{M}$ and with $1+\underline{P}_{M}$, respectively

The same technique can be applied to establish such a valid lower bound for the trajectory on the intervals $(d e c, 1)$ and $(i n c, n)$, that can be applied for further bound improvements even in the case when the necessary integration should start from the $z_{2}$ zero.

Let us see now how can we produce stronger bounds on the intervals $(\operatorname{dec}, n)$ and (inc, $n$ ) before the $z_{1}-1$, and $z_{2}-1$ time points, respectively - on the basis of the bounds discussed earlier in the present subsection. Consider first the $($ dec,$n)$ case, then for the present upper bounding function

$$
y_{(\text {dec }, n)}^{(\text {upper })} \geq y(t) .
$$

Integrate the derivative function $y^{\prime}$ from $t$ to $z_{1}$, where $z_{1}-1 \leq t \leq z_{1}$ :

$$
-y(t)=y\left(z_{1}\right)-y(t)=-\alpha \int_{t}^{z_{1}} e^{y(x-1)}-1 d x=-\alpha \int_{t-1}^{z_{1}-1} e^{y(x)}-1 d x .
$$

In other terms

$$
y(t) \leq \alpha \int_{t-1}^{z_{1}-1} e^{y_{(\text {dec }, n)}^{(\text {uppr })}(x)}-1 d x
$$


This bounding function can be use to update the old one:

$y_{(\text {dec }, n)}^{(\text {upper })}(t)=\min \left\{\begin{array}{c}y_{(\text {dec }, n)}^{(\text {upper })}(t) \\ \alpha \int_{t-1}^{z_{1}-1} e^{y_{(\text {dec }, n)}^{(\text {upper })}(x)}-1 d x\end{array}\right\}$ if $t \in\left[z_{1}-1, z_{1}\right]$.

In a similar way we can calculate a new lower bounding function on the interval (inc, $n)$ :

$$
y(t) \geq \alpha \int_{t-1}^{z_{2}-1} e^{y_{(\text {inc }, n)}^{(\text {low })}}-1 d x
$$

that implies the update

$y_{(\text {inc }, n)}^{(\text {lower })}(t)=\min \left\{\begin{array}{c}y_{(\text {inc }, n)}^{(\text {lower })}(t) \\ \alpha \int_{t-1}^{z_{2}-1} e^{y_{(\text {inc }, n)}^{(\text {lower })}(x)}-1 d x\end{array}\right\}$ if $t \in\left[z_{2}-1, z_{2}\right]$.

Notice that in both cases the new, improved bound utilizes earlier bound values also from more than 1 time unit distance to the actual right end zero of the trajectory. This gives an explanation how improvements made at the first part of the present subsection can improve our bounds at a much later time point.

\section{The iterative improvement of the bounding functions}

The lower and upper bounds derived in the earlier subsections will be applied in an iterative procedure to make them even sharper that possibly allows to conclude that for a given pair of $M$ and $m$ values the delay differential equation (1) with the investigated interval of $\alpha$ parameter leads to a contradiction. The iteration cycle begins with the time interval (inc, 1$)$, and with the integration of the right hand side of the differential equation we update the earlier upper bound on $(\mathrm{dec}, n)$. This new upper bound will then be used to improve the lower and upper bounding functions on $($ dec, 1$)$, and finally the latter help us to make $y_{(i n c, n)}^{(l o w e r)}$ sharper.

Now the bounding functions $y_{(\text {inc }, 1)}^{(\text {lower })}, y_{(\text {dec }, 1)}^{(\text {upper })}, y_{(i n c, 1)}^{(\text {upper })}$, and $y_{(\text {dec }, 1)}^{(\text {lower })}$ are defined on unit length time intervals, on $[0,1]$ and $\left[z_{1}, z_{1}+1\right]$, respectively. In contrast to these, in the case of $y_{(\text {inc }, n)}^{(\text {lower })}$ and $y_{(\text {dec }, n)}^{(\text {upper })}$ we must also calculate with their values over wider time intervals. To be able to handle the delayed terms, we have to save bounding function values for a unit length interval in the first case, and for two width intervals otherwise (this later figure proved to be satisfactory for our investigation).

Due to the computer representation of reals, it is advantageous to subdivide these time intervals into $2^{l}$, and $2^{l+1}$ subintervals for a natural number $l$, respectively. 
Denote these subintervals by $t_{i}$, where $i \in\left(1, \ldots, 2^{l}\right)$, and for the $($ dec,$n)$ and $($ inc,$n)$ time intervals $i \in\left(1, \ldots, 2^{l+1}\right)$ in increasing order as they depart from the zero. It is intentional that the order of the numeration for the unit length intervals is the opposite of that for $(\mathrm{dec}, n)$ and $(i n c, n)$. Within such a subinterval, the respective bounding function will be represented by a real number, i.e. we use a bounding step function for the saved bounding functions. This step function is denoted by $Y$, as also in Section 4. The right hand side of the differential equation can then easily be bounded using the step functions both at $t_{j}$ and at the same time at $t_{j}-1$. The updated value of $Y_{(\text {inc, }, 1)}^{(\text {upper })}\left(t_{i}\right)\left(i=1, \ldots, 2^{l}\right)$ can be calculated applying $Y_{(\text {inc }, n)}^{(\text {lower })}$ according to (7):

$Y_{(\text {inc }, 1)}^{(\text {upper })}\left(t_{i}\right)=\min \left\{-\alpha \sum_{j=1}^{i}\left(e^{Y_{(\text {inc }, n)}^{(\text {lower })}\left(t_{2 l}{ }_{-j+1}\right)}-1\right) / 2^{l} ; Y_{(\text {inc }, 1)}^{(\text {upper })}\left(t_{i}\right)\right\}$.

In a similar way, we can obtain the other bounding functions updated using the stronger bounds given as (8) to (10):

$$
\begin{aligned}
& Y_{(\text {dec }, 1)}^{(\text {lower })}\left(t_{i}\right)=\max \left\{-\alpha \sum_{j=1}^{i}\left(e^{Y_{(\text {dec }, n)}^{(\text {upper })}\left(t_{2^{l}-j+1}\right)}-1\right) / 2^{l} ; Y_{(\text {dec }, 1)}^{(\text {lower })}\left(t_{i}\right)\right\}, \\
& Y_{(\text {inc }, 1)}^{(\text {lower })}\left(t_{i}\right)=\max \left\{M+\alpha \sum_{j=i}^{2^{l}}\left(e^{Y_{(\text {inc }, n)}^{(\text {lower })}\left(t_{2^{l}-j+1}\right)}-1\right) / 2^{l} ; Y_{(\text {inc }, 1)}^{(\text {lower })}\left(t_{i}\right)\right\} \text {, } \\
& \left.Y_{(\text {dec }, 1)}^{(\text {upper })}\left(t_{i}\right)=\min \left\{-m+\alpha \sum_{j=i}^{2^{l}}\left(e^{Y_{(\text {dec }, n)}^{(\text {upper })}\left(t_{2} l-j+1\right.}\right)-1\right) / 2^{l} ; Y_{(\text {dec }, 1)}^{(\text {upper })}\left(t_{i}\right)\right\} \text {. }
\end{aligned}
$$

On the basis of these bounding functions, we can calculate bounds on the trajectory for the next, not unit length time intervals. The bounds on the trajectory will provide lower and upper bounds on the next zero, as discussed in Section 4. Thus we obtain lower and upper bounds on the trajectory on the time intervals $\left[0,1+\underline{P}_{M}\right]$, and $[0,1+$ $\left.\bar{P}_{M}\right]$, respectively. The formal description of the algorithm for the determination of the bounds of zeros is given as Algorithm 1. Here we bound the trajectory after the time 1 , or $z_{1}+1$, and check whether the respective $Y\left(t_{j}\right)$ interval contains zero. The algorithm is able to identify lower and upper bounds within length 2 intervals, this was satisfactory for our investigation. The reordering of the $2^{-l}$ size subintervals mentioned in Section 7 must be made after Algorithm 1 was run.

Consider now how these bounding functions can be used to improve $y_{(\text {dec }, n)}^{(\text {upper })}$. The integration of the step function $Y\left(t_{i}\right), i \in\left(1, \ldots, 2^{l}\right)$ gives with (11) and (12) the updated upper and lower bounding functions

$$
Y_{(\text {dec }, n)}^{(\text {upper })}\left(t_{i}\right)=\max \left\{\alpha \sum_{j=i}^{2^{l}}\left(e^{Y_{(\text {dec }, n)}^{(\text {upper })}\left(t_{j-2} l\right)}-1\right) / 2^{l} ; Y_{(\text {dec }, n)}^{(\text {upper })}\left(t_{i}\right)\right\}
$$




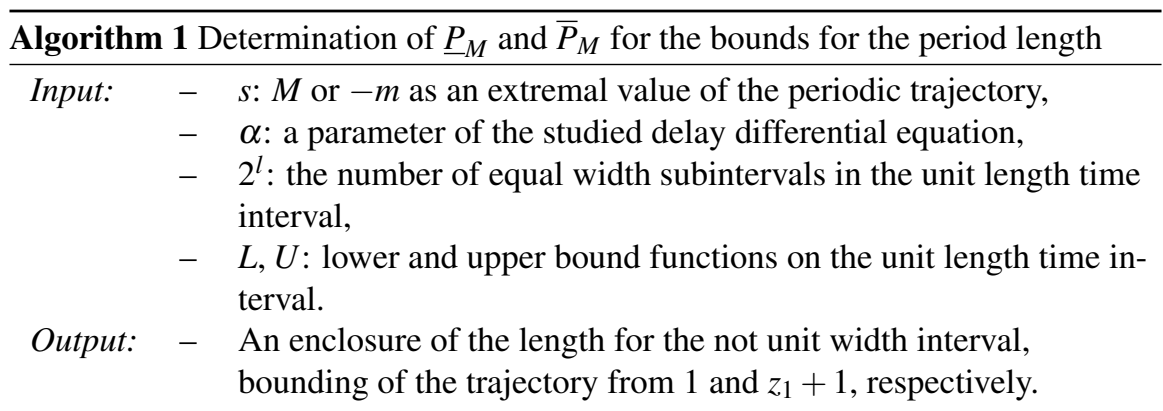

Step 1. Compute $Y\left(t_{i}\right)\left(i=1, \ldots, 2^{l}\right)$ as the enclosures of the periodic solution on subintervals of the unit length time period by using the $U$ and $L$ functions on the $($ inc, 1$)$ and $($ dec, 1$)$ intervals.

Step 2. Set $j=\left(2^{l}+1\right)$ and $Y_{\text {last }}=[s, s]$.

Step 3. Enclose $Y\left(t_{j}\right)$ with the expression $\left(Y_{\text {last }}+\left(-\alpha\left(e^{Y\left(t_{j-2^{l}}\right)}-1\right)\right) \cdot\left[0,1 / 2^{l}\right]\right)$.

Step 4. Set $Y_{\text {last }}=Y_{\text {last }}+\left(-\alpha\left(e^{Y\left(t_{j-2} l\right)}-1\right)\right) / 2^{l}$.

Step 5. If $0 \notin Y\left(t_{j-1}\right)$ and $0 \in Y\left(t_{j}\right)$, then calculate the new lower bound for the length of the not unit width interval: $\underline{P}_{M}=(j-1) / 2^{l}$.

Step 6. If $0 \in Y\left(t_{j-1}\right)$ and $0 \notin Y\left(t_{j}\right)$, then calculate the new upper bound for the length of the not unit width interval: $\bar{P}_{M}=(j-1) / 2^{l}$ and STOP.

Step 7. Set $j=j+1$.

Step 8. If $j<2^{l+2}$, then continue with Step 3, otherwise STOP.

and

$Y_{(\text {inc }, n)}^{(\text {lower })}\left(t_{i}\right)=\min \left\{\alpha \sum_{j=i}^{2^{l}}\left(e^{Y_{(\text {inc }, n)}^{(\text {lowe })}\left(t_{j-2^{l}}\right)}-1\right) / 2^{l} ; Y_{(\text {inc }, n)}^{(\text {lower })}\left(t_{i}\right)\right\}$.

This completes the description of the iterative procedure to improve bounding functions on the periodic solutions of the delay differential equation (1). The periodic solution should reach at the time point 1 the maximal value of $M$, while at the end of $(d e c, 1)$ the value $-m$. We can use this fact as a condition to be checked, whether to the given $M, m$ pair a periodic solution belongs for the actual $\alpha$ differential equation parameter. The corresponding inequalities are (cf. (6)):

$$
Y_{(\text {inc }, 1)}^{(\text {upper })}\left(t_{2^{n}}\right) \geq M \quad \text { and } \quad Y_{(\text {dec }, 1)}^{(\text {lower })}\left(t_{2^{n}}\right) \leq-m
$$

The checking algorithm is also able to decide on these conditions when the $M$ values are given as intervals. To exclude such possible intervals of $M$ we apply the above conditions for the upper bounds of the respective intervals:

$Y_{(\text {inc }, 1)}^{(\text {upper })}\left(t_{2^{n}}\right)<\underline{M}$.

By this condition we can delete all points of the respective subintervals. 


\section{Extension of the parameter range}

In [2] the Wright conjecture was proven for $\alpha$ values between 1.5 and 1.5706 . We continued the computational part of the proof with unchanged theoretical background. The computational environment was a blade server with 12 cores and 24 threads, we set the algorithm parameters in the same way for all checked new subintervals. In this way, the computation times in Table 1 reflect well the necessary increasing computational complexity.

Table 1

The CPU time requirements of the proven $\alpha$ intervals.

\begin{tabular}{cc}
\hline Interval & CPU time in hours \\
\hline \hline$[1.57060,1.57061]$ & 56.9 \\
{$[1.57061,1.57062]$} & 64.9 \\
{$[1.57062,1.57063]$} & 83.7 \\
{$[1.57063,1.57064]$} & 119.4 \\
{$[1.57064,1.57065]$} & 141.2 \\
\hline
\end{tabular}

Seeing the data in Table 1 we can draw the conclusion that the necessary computation times for proving new subintervals with unchanged algorithm parameters grows in a highly nonlinear way. That confirms our earlier conclusion drawn in [2] that additional theoretical insight should be utilized to achieve a substantial progress in the proven $\alpha$ values. After submitting our manuscript, J. Bouwe van den Berg and J. Jaquette published their theoretical proof on the remaining part of Wright's conjecture [3], that was based on our earlier computational result [2]. It confirms indirectly, that our bounding scheme approach is justified for the larger part of the $\alpha$ parameter interval in the conjecture.

Acknowledgement. The research work was partially supported by the EFOP-3.6.3VEKOP-16-2017-00002 project "Integrated program for young research associates in the fields of informatics and computer science". The authors are grateful for the coding and testing support given by Zsolt Bagóczki.

\section{References}

[1] B. BÁNHELYI, The investigation of a delay differential equation by a verified computer procedure (in Hungarian), Alkalmazott Matematikai Lapok 24(2007) 131-150.

[2] B. Bánhelyi, T. Csendes, T. Krisztin, And A. Neumaier, Global attractivity of the zero solution for Wright's equation, SIAM J. on Applied Dynamical Systems 13(2014) 537-563.

[3] J. BouWe VAN DEN BERG AND J. JAQUETTE, A proof of Wright's conjecture. arXiv: 1704.00029 . 
[4] N.S. Nedialkov, K.R. Jackson, And G.F. Corliss, Validated Solutions of Initial Value Problems for Ordinary Differential Equations, Applied Mathematics and Computation, 105(1999) 21-68.

[5] H. RATSCHEK AND J. RoKNE, Computer Methods for the Range of Functions. Ellis Horwood, Chichester, 1984.

[6] E.M. WRIGHT, A non-linear difference-differential equation, J. für die Reine und Angewandte Mathematik 194 (1955) 66-87. 